\title{
EFICÁCIA DE INTERVENÇÕES EDUCATIVAS COM GESTANTES SOBRE O GRAU DE CONHECIMENTO EM ALEITAMENTO MATERNO
}

\section{Effectiveness of educational interventions with pregnant women on the level of knowledge about breastfeeding}

\section{Eficacia de intervenciones educativas con embarazadas sobre el grado de conocimiento de la lactancia materna}

\author{
Andreza Kathiuze Maia (iD \\ Instituto Federal de Educação, Ciência e Tecnologia do Ceará - IFCE - Limoeiro do Norte (CE) - Brasil \\ Bruna Yhang da Costa Silva iD \\ Instituto Federal de Educação, Ciência e Tecnologia do Ceará - IFCE - Limoeiro do Norte (CE) - Brasil \\ Luis Clenio Jário Moreira iD \\ Instituto Federal de Educação, Ciência e Tecnologia do Ceará - IFCE - Limoeiro do Norte (CE) - Brasil
}

\section{RESUMO}

Objetivo: Avaliar o grau de conhecimento sobre aleitamento materno de mulheres na primeira metade gestacional em pré-natal pelo Sistema Único de Saúde (SUS) e desenvolver atividades de educação em saúde sobre essa temática visando melhorar o nível de conhecimento das gestantes. Métodos: Estudo longitudinal, de intervenção, realizado nas Unidades de Atenção Primária à Saúde (UAPS) de Limoeiro do Norte, Ceará, Brasil, com 57 mulheres na primeira metade gestacional. Foram coletadas informações socioeconômicas sobre escolaridade, etnia, atividade profissional, renda familiar e situação conjugal, e realizada avaliação do grau de conhecimento acerca de quatro blocos temáticos relacionados ao aleitamento materno: cuidados pré-parto com as mamas, vantagens da amamentação para o binômio mãe-filho, fisiologia da lactação e técnicas adequadas de amamentação. Posteriormente, foram realizadas quatro intervenções educativas, na frequência de uma por semana, nas UAPS ou em domicílio, com enfoque nas quatro abordagens do formulário. O instrumento de avaliação de conhecimento foi, então, reaplicado. Resultados: O grau de conhecimento das gestantes melhorou em todos os domínios do aleitamento materno investigados: de $62,8 \%$ de acertos para $83,0 \%$ no bloco temático um (variação: $20,2 \%$ ); de $71,6 \%$ para $87,5 \%$ no bloco dois (15,9\%); de $66,9 \%$ para $79,7 \%$ no três $(12,7 \%)$; de $63,6 \%$ para $92,6 \%$ no bloco quatro $(29,0 \%)$. Observou-se maior impacto sobre o aspecto "técnicas adequadas de amamentação". Conclusão: As gestantes avaliadas apresentaram conhecimento adequado sobre a maioria dos questionamentos acerca do aleitamento materno. A educação em saúde foi eficaz em melhorar esses conhecimentos, o que futuramente poderá refletir no prolongamento do tempo de amamentação exclusiva.

Descritores: Aleitamento Materno; Educação em Saúde; Gravidez.

Número de Registro (ReBEC): RBR-3rs7sx

\section{ABSTRACT}

Objective: To evaluate the level of knowledge about breastfeeding in women in the first half of pregnancy receiving prenatal care through the Unified Health System (Sistema Único de Saúde - SUS) and carry out health education activities on this subject with the aim of improving pregnant women's level of knowledge. Methods: This longitudinal intervention study was conducted at the Primary Health Care (PHC) centers of Limoeiro do Norte, Ceará, Brazil with 57 women in the first half of pregnancy. Socioeconomic information on education, ethnicity, professional activity, household income, and marital status was collected and knowledge of four breastfeeding-related thematic blocks was evaluated: prenatal breast care, advantages of breastfeeding for the mother-child binomial. child, lactation physiology and appropriate breastfeeding techniques. Subsequently, four educational interventions were carried out on a weekly basis either in the PHC centers or at home focusing on the four approaches described in the form. The knowledge evaluation instrument was then reapplied. Results: Pregnant women's level of knowledge improved in all the domains of breastfeeding analyzed: from $62.8 \%$ to $83.0 \%$ of correct answers in thematic block one (range: $20.2 \%$ ); from $71.6 \%$ to $87.5 \%$ in block two (15.9\%); from $66.9 \%$ to $79.7 \%$ in block three (12.7\%); from $63.6 \%$ to $92.6 \%$ in block four (29.0\%). There was a greater impact on "adequate breastfeeding techniques". Conclusion: Pregnant women had adequate knowledge about 
most questions about breastfeeding. Health education was effective in improving such knowledge, which in the future may reflect on the prolongation of exclusive breastfeeding duration.

Descriptors: Breastfeeding; Health Education; Pregnancy

Registration Number (ReBEC): RBR-3rs7sx

\section{RESUMEN}

Objetivo: Evaluar el grado de conocimiento sobre la lactancia materna de mujeres en la primera mitad del embarazo y en el prenatal por el Sistema Único de Salud (SUS) y desarrollar actividades de educación en salud sobre el tema para mejorar el nivel de conocimiento de las embarazadas. Métodos: Estudio longitudinal y de intervención realizado en las Unidades de Atención Primaria de Salud (UAPS) de Limoeiro do Norte, Ceará, Brasil, con 57 mujeres en la primera mitad del embarazo. Se recogieron las informaciones socioeconómicas sobre la escolaridad, la etnia, la actividad profesional, la renta familiar y la situación conyugal y realizada la evaluación del grado de conocimiento sobre los cuatro bloques temáticos relacionados con la lactancia materna: los cuidados preparto con las mamas, las ventajas de la lactancia materna para el binomio madre-hijo, la fisiología de la lactación y las técnicas adecuadas de lactancia. Se han realizado, a posteriori, cuatro intervenciones educativas una vez a la semana en las UAPS o en el domicilio con énfasis en los cuatro abordajes del formulario. El instrumento de evaluación de conocimiento ha sido reaplicado. Resultados: El grado de conocimiento de las embarazadas ha mejorado en todos los dominios de la lactancia materna que han sido investigados: del 62,8\% de aciertos para el 83,0\% del bloque temático uno (variación: 20,2\%); del 71,6\% para el $87,5 \%$ del bloque dos (15,9\%); del 66,9\% para el 79,7\% del tres (12,7\%); del 63,6\% para el 92,6\% del bloque cuatro (29,0\%). Se ha observado mayor impacto en el aspecto "técnicas adecuadas de lactancia". Conclusión: Las embarazadas evaluadas presentaron conocimiento adecuado sobre la mayoría de las preguntas acerca de la lactancia materna. La educación en salud ha sido eficaz para la mejoría de los conocimientos lo que en el futuro podrá reflejar en el aumento del tiempo de lactancia materna.

Descriptores: Lactancia Materna; Educación en Salud; Embarazo.

\section{INTRODUÇÃO}

As necessidades de saúde de uma população estão em constante mudança. Ao pensar nessas necessidades no âmbito da saúde física, imediatamente lembra-se da procura por assistência, que consiste, principalmente, na busca por informações de saúde. Na atenção primária, comumente a propagação de informações é falha e ocasiona prejuízos, como a menor motivação das mulheres para o processo da amamentação(1).

Com isso, observa-se em vários países o uso de práticas inadequadas, como desmame precoce e a realização de complementação alimentar de forma equivocada. Essas práticas podem se associar à má nutrição em lactentes e também a problemas relacionados ao crescimento, desenvolvimento e mortalidade nessa faixa etária(2). Dessa forma, precisam ser reforçadas as informações referentes às vantagens do aleitamento materno exclusivo, assim como os potenciais prejuízos do desmame precoce ${ }^{(3)}$.

$\mathrm{O}$ aleitamento materno traz benefícios tanto para a saúde da mulher quanto da criança envolvida ${ }^{(3)}$. O leite materno exclusivo até o sexto mês de vida nutre o lactente de forma completa e, assim, promove crescimento e desenvolvimento adequados e fornece suporte imunológico sistêmico, que se traduz, por exemplo, na redução do risco de desenvolvimento de gastrenterite, doenças respiratórias, diabetes tipos 1 e 2, obesidade e leucemia(4).

Na saúde da mulher, citam-se benefícios como a prevenção de cânceres de mama e de ovário, obesidade, diabetes melitus e hipertensão arterial. Outro ganho inclui a praticidade de ter o alimento pronto, o que evita despesas com substitutos do leite materno ${ }^{(3)}$.

Desse modo, a prática do aleitamento materno é estratégia eficaz na redução da mortalidade infantil, principalmente pela ação preventiva da diarreia e de infecções respiratórias, pelo menos em países em desenvolvimento, com resultados incertos em países desenvolvidos quanto à taxa de mortes ${ }^{(4)}$. Mesmo com todas essas evidências, percebe-se que são vários os aspectos que induzem as mães a não amamentarem ${ }^{(5)}$ ou a realizarem desmame precoce ${ }^{(2)}$. Nas capitais brasileiras, por exemplo, a prevalência de aleitamento materno exclusivo (AME) em lactentes com menos de seis meses é de apenas $41 \%$. Os piores resultados são percebidos no Norte $(45,9 \%)$ e no Nordeste do país ${ }^{(5)}$.

A informação e a motivação são dois determinantes da concretização do aleitamento materno. No percurso entre o desejo e o sucesso da prática, a motivação é o que permeia a decisão materna, de modo favorável ou contrário, embora fatores sociais também interfiram ${ }^{(1)}$. É relevante perceber a assistência pré-natal como um momento oportuno para dialogar com as gestantes sobre sua realidade e seu desejo de amamentar. Além disso, uma vez que o desmame precoce é um problema de saúde pública, é necessário que os profissionais de saúde, 
nos diversos níveis de atendimento, estabeleçam práticas educativas direcionadas à amamentação, a fim de intervir no tempo de desmame ${ }^{(6)}$.

A Política Nacional de Promoção da Saúde (PNPS) estabelece que a educação permanente de trabalhadores da saúde e o desenvolvimento de ações de promoção da saúde para a população devam ser temas prioritários nos diálogos e ações governamentais e não governamentais ${ }^{(7)}$. Contudo, na prática, percebe-se que as equipes de Atenção Primária à Saúde (UAPS) apresentam metas exacerbadas de número de atendimentos, ausência de espaço físico adequado e carência de recursos materiais básicos, o que dificulta a priorização da prática educativa voltada para a promoção da saúde, inclusive do aleitamento materno exclusivo ${ }^{(8)}$, pelos profissionais.

Assim, este trabalho teve como objetivo avaliar o grau de conhecimento sobre aleitamento materno de mulheres na primeira metade gestacional em pré-natal pelo Sistema Único de Saúde e desenvolver atividades de educação em saúde sobre essa temática, visando melhorar o nível de conhecimento das gestantes.

\section{MÉTODOS}

Trata-se de um estudo longitudinal, de intervenção, realizado em dez UAPS de Limoeiro do Norte, Ceará, Brasil, com mulheres na primeira metade gestacional.

A população consistiu em 182 gestantes que, em janeiro de 2018, realizavam pré-natal em alguma das UAPS de Limoeiro do Norte. A amostra foi calculada usando população finita de 182 gestantes, considerando-se o intervalo de confiança de $95 \%$ e erro amostral de $5 \%^{\left({ }^{(9)}\right.}$, o que resultou em 50 mulheres.

Por se prever possíveis desistências, em virtude de ser um estudo com seguimento, foram incluídas na pesquisa, além das 50, mais sete mulheres, que atenderam aos seguintes critérios: ser gestante em idade adulta, isto é, a partir de $19 \operatorname{anos}^{(10)}$, e que estivesse na primeira metade da gestação, ou seja, com ou até 20 semanas de gestação. Adotou-se como critério de exclusão a ausência de alfabetização ou a presença de déficits cognitivos que inviabilizassem a reposta ao instrumento de coleta.

Inicialmente, realizaram-se visitas nas UAPS para conversar com as gestantes sobre o objetivo e os procedimentos do estudo, sendo perguntado sobre o interesse delas em participarem. Após o aceite, as mulheres leram e assinaram um Termo de Consentimento Livre e Pós-Esclarecido (TCLE). A abordagem ocorreu conforme a metodologia resumida na Figura 1.

Assim, na primeira etapa da coleta de dados, as gestantes responderam a um formulário socioeconômico que interrogava sobre escolaridade, etnia, atividade profissional, renda familiar e situação conjugal, bem como 30 perguntas fechadas, com opções de resposta "sim" ou "não", que avaliava seus conhecimentos acerca do aleitamento materno e seus determinantes ${ }^{(11)}$. As perguntas foram agrupadas em quatro blocos temáticos: conhecimentos das mães sobre o preparo das mamas antes do parto; vantagens da amamentação para o bebê e para a mãe; fisiologia da lactação; e técnicas adequadas de amamentação. O tempo de aplicação do instrumento variou em torno de 12 a 15 minutos para cada participante.

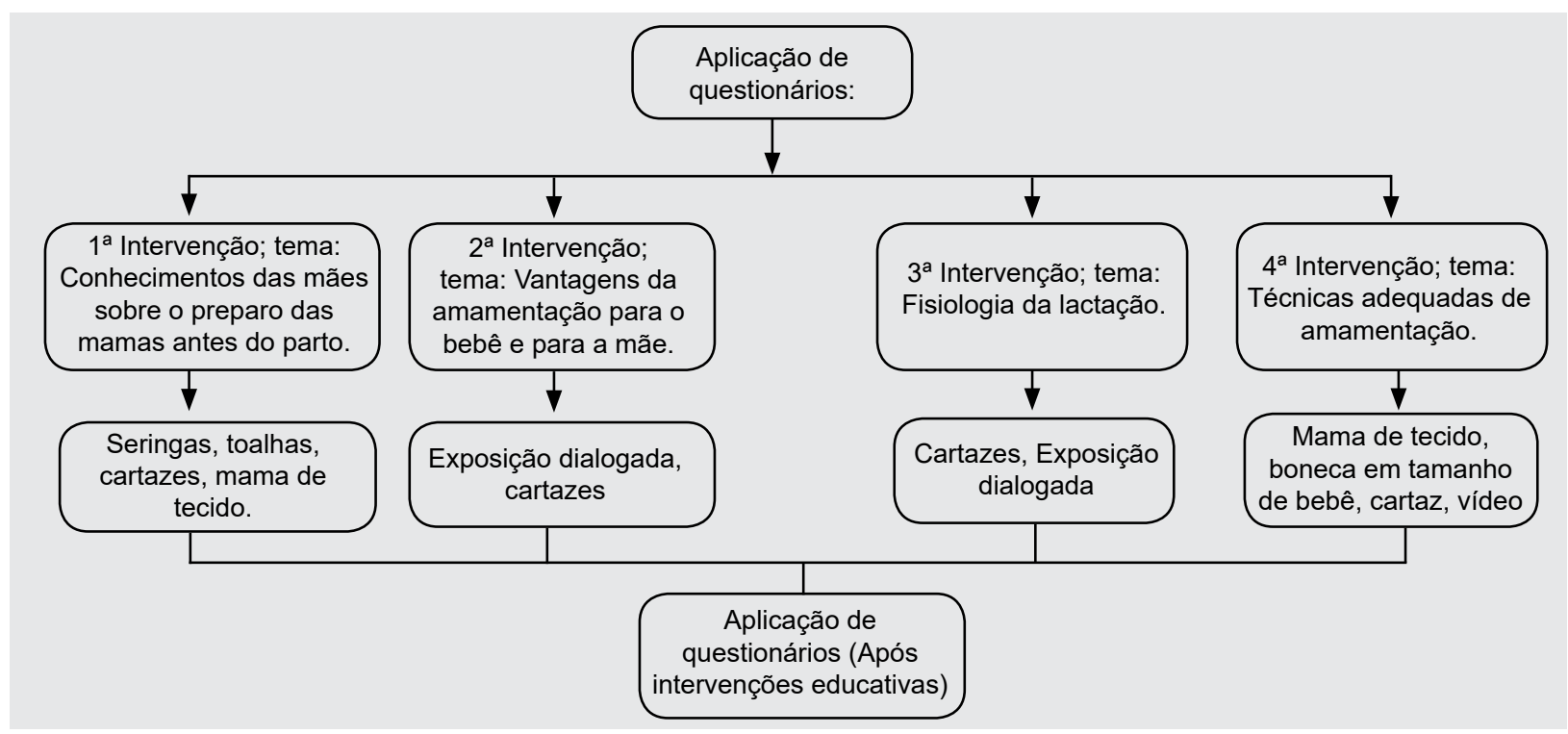

Figura 1 - Fluxograma da metodologia utilizada. 
Em seguida, os encontros, agendados em cada uma das UAPS, no turno da manhã, ocorreram nos respectivos dias reservados ao atendimento de gestantes para a realização da intervenção educativa. As datas das atividades eram comunicadas no dia de aplicação dos questionários e relembradas pelas agentes comunitárias de saúde (ACS). Na ausência das gestantes em algum dos momentos, realizaram-se visitas domiciliares com o auxílio das agentes comunitárias de saúde.

A intervenção teve quatro momentos, na frequência de um por semana, cada um referente a um bloco temático do formulário. A metodologia de intervenção utilizada consistiu na elaboração de cartazes a serem utilizados como recurso educativo nas exposições dialogadas, os quais foram afixados nos flanelógrafos das UAPS. Produziu-se uma mama de tecido para demonstração da técnica de ordenha manual e dos tipos de mamilos e sua relação com a amamentação. Para a elaboração dos recursos educativos, utilizou-se referências de importância na área de nutrição materno-infantil, com foco específico em aleitamento materno e/ou em estratégias a serem adotadas no processo de ensino-aprendizagem crítico-reflexivo sobre essa temática ${ }^{(12-15)}$. Apresentou-se, ainda, um vídeo de autoria do Ministério da Saúde, em parceria com a Sociedade Brasileira de Pediatria ${ }^{(16)}$, que resumia a maioria dos questionamentos abordados no formulário.

Após cada dia de atividade, as mulheres eram questionadas acerca de possíveis dúvidas, para pronta resolução. Por fim, houve a reaplicação do formulário de avaliação prévia de conhecimentos ${ }^{(11)}$ para avaliação dos conhecimentos pós-intervenção.

Realizou-se a tabulação dos dados em planilha do Excel, na qual foram listadas todas as gestantes, seus respectivos dados socioeconômicos e número de perguntas acertadas acerca de cada abordagem, antes e após a intervenção.

Os dados socioeconômicos foram apresentados em frequência absoluta e percentual. Para apresentação dos resultados da avaliação de conhecimentos, determinou-se a média de acertos do grupo de gestantes por temática, antes e após intervenção, para comparação.

Utilizou-se o teste $\mathrm{t}$ de Student para verificar a diferença na média percentual de acertos antes e depois da intervenção. Adotou-se $p<0,05$ como nível de significância.

Realizou-se a pesquisa somente após sua aprovação pelo Comitê de Ética em Pesquisa do Instituto Federal de Educação, Ciência e Tecnologia do Ceará (IFCE), com o Parecer $n .^{\circ}$ 2.457.636. A pesquisa foi registrada na plataforma brasileira de ensaios clínicos, obtendo o número de registro (ReBEC): RBR-3rs7sx.

\section{RESULTADOS}

Participaram da $1^{\text {a }}$ etapa (avaliação de conhecimento das gestantes) um total de 57 mulheres. Contudo cinco $(8,8 \%)$ gestantes não completaram as quatro abordagens educativas, pois duas $(3,5 \%)$ mudaram de município, uma $(1,8 \%)$ não informou no questionário nem endereço nem telefone, e esses dados também não eram conhecidos pelas ACS, e as outras duas (3,5\%) não foram encontradas no endereço fornecido, inviabilizando a continuidade no estudo. Desse modo, resultaram $52(91,2 \%)$ mulheres contempladas com todas as etapas da pesquisa.

A Tabela I mostra a distribuição das gestantes de acordo com as variáveis socioeconômicas.

As mulheres tinham em média 27 anos, variando de 19 a 39 anos. Vê-se que predominou entre as participantes gestantes com ensino médio completo $(40,4 \% ; n=21)$, pardas $(56,0 \% ; n=28)$, com renda familiar inferior a um salário mínimo $(48,9 \% ; n=23)$ e casadas $(70,6 \% ; n=36)$.

A Tabela II, a seguir, apresenta o grau de conhecimento das gestantes sobre aleitamento materno conforme temáticas de abordagem, antes e após a intervenção educativa.

Vê-se que o grau de conhecimento prévio das gestantes sobre aleitamento materno foi superior a $50 \%$ em todas as abordagens do tema, com maior destaque para "as vantagens da amamentação para o bebê" (média de 71,6\% de respostas corretas). Após as atividades educativas, o conhecimento melhorou em todos os blocos temáticos, o que correspondeu a uma média de $20,2 \%$ de acréscimo nos acertos acerca do "preparo das mamas antes do parto", $15,9 \%$ de melhoria para o tema "vantagens da amamentação para o bebê e para a mãe", 12,7\% para "a fisiologia da lactação" e 29,0\% para "as técnicas adequadas de amamentação". Ao final, a média de conhecimento das gestantes foi superior na abordagem voltada para as técnicas adequadas de amamentação. 
Tabela I - Caracterização de dados socioeconômicos das gestantes de Limoeiro do Norte, Ceará, 2018 ( $\mathrm{n}=$ 57).

\begin{tabular}{|c|c|c|}
\hline Variáveis & $\mathbf{n}$ & $(\%)$ \\
\hline \multicolumn{3}{|l|}{ Escolaridade } \\
\hline Analfabeto & 1 & 1,9 \\
\hline Ens. Fund.amental incompleto & 8 & 15,4 \\
\hline Ens. Fundamental completo & 2 & 3,8 \\
\hline Ens. médio incompleto & 7 & 13,5 \\
\hline Ens. médio completo & 21 & 40,4 \\
\hline Ens. superior incompleto & 9 & 17,3 \\
\hline Ens. superior completo & 4 & 7,7 \\
\hline TOTAL & $52^{*}$ & 100,0 \\
\hline \multicolumn{3}{|l|}{ Etnia } \\
\hline Branco & 17 & 34,0 \\
\hline Negro & 3 & 6,0 \\
\hline Pardo & 28 & 56,0 \\
\hline Amarelo & 2 & 4,0 \\
\hline TOTAL & $50^{*}$ & 100,0 \\
\hline \multicolumn{3}{|l|}{ Renda Familiar } \\
\hline Menor que um salário mínimo & 23 & 48,9 \\
\hline De um a três salários mínimos & 20 & 42,5 \\
\hline De quatro a seis salários mínimos & 2 & 4,3 \\
\hline Maior que seis salários mínimos & 2 & 4,3 \\
\hline TOTAL & $47^{*}$ & 100,0 \\
\hline \multicolumn{3}{|l|}{ Situação Conjugal } \\
\hline Solteira & 14 & 27,4 \\
\hline Casada & 36 & 70,6 \\
\hline Divorciada & 1 & 2,0 \\
\hline TOTAL & $51^{*}$ & 100,0 \\
\hline
\end{tabular}

*: O total diferiu entre as variáveis, pois algumas mulheres deixaram perguntas sem resposta. Fund: fundamental; Ens: ensino

Tabela II - Percentual de acertos das gestantes antes e depois das intervenções educativas, dividido por grupos de perguntas, Limoeiro do Norte, Ceará, $2018(n=57)$.

\begin{tabular}{|c|c|c|c|c|}
\hline Abordagens & $\begin{array}{c}\text { Antes } \\
(\%)\end{array}$ & $\begin{array}{l}\text { Após } \\
(\%)\end{array}$ & $\begin{array}{c}\text { Acréscimo nos } \\
\text { acertos }(\%)\end{array}$ & $P$-value \\
\hline 1) Preparo das mamas antes do parto & 62,8 & 83,0 & 20,2 & $<0,001$ \\
\hline 2) Vantagens da amamentação para o bebê e para a mãe & 71,6 & 87,5 & 15,9 & $<0,001$ \\
\hline 3) Fisiologia da lactação & 66,9 & 79,7 & 12,7 & $<0,001$ \\
\hline 4) Técnicas adequadas de amamentação & 63,6 & 92,6 & 29,0 & $<0,001$ \\
\hline
\end{tabular}

*: teste t de Student $\mathrm{p}<0,05$ como significante

\section{DISCUSSÃO}

No presente estudo, as gestantes apresentaram conhecimento adequado sobre a maioria dos questionamentos realizados acerca do aleitamento materno. Além disso, as intervenções sobre os temas, por meio de educação em saúde, foram eficazes em melhorar esses conhecimentos.

Essa temática é de fundamental importância, uma vez que, de acordo com dados do ano de 2008, a prevalência de aleitamento materno exclusivo no Brasil, em menores de seis meses de idade, era de $41 \%{ }^{(5)}$, quando essa prática deveria ser regra até o sexto mês de vida ${ }^{(17,18)}$.

Embora não tenha sido objetivo desta pesquisa relacionar fatores socioeconômicos ao nível de conhecimento sobre aleitamento materno ou com a prática da amamentação em si, é importante destacar que a média de idade das gestantes desta pesquisa (27 anos) está inserida, quando se trata de região Nordeste do Brasil, em uma faixa etária (20 a 29 anos) cuja mediana de duração do aleitamento materno estimada é 183 dias. Para nutrizes com idade de 15 a 19 anos essa mediana parece ser de 159,3 dias e, para aquelas com mais de 30 anos, 265,5 dias ${ }^{(19)}$. 
Esses dados sugerem que a duração do aleitamento materno é diretamente proporcional à idade das nutrizes, mas inferior à recomendação de dois anos ou mais de amamentação, propagada pela Organização Mundial de Saúde desde 2002, ou de, pelo menos, 12 meses, segundo a Academia Americana de Pediatria(17,18).

A escolaridade predominante entre as gestantes foi alta (ensino médio completo), ao considerar que o fundamental incompleto predomina na população brasileira ${ }^{(20)}$. Esse achado é favorável, uma vez que parece existir relação direta entre anos de estudo e melhores cuidados maternos com os filhos, nos quais pode-se incluir o tempo adequado de aleitamento materno e a prática do aleitamento materno exclusivo ${ }^{(21)}$.

A situação conjugal de maior prevalência entre as entrevistadas (casadas) pode impactar positivamente, pois a existência de uma estrutura familiar confortável e a presença do cônjuge no lar como sujeito incentivador da prática do aleitamento materno são determinantes para maior tempo de permanência do binômio mãe-filho no processo ${ }^{(22)}$.

Em relação aos achados anteriores às intervenções, houve um percentual de erro nos questionamentos superior a $28 \%$ em todas as abordagens, com as perguntas acerca do preparo das mamas antes do parto se destacando com maior percentual de respostas equivocadas $(37,2 \%)$, seguidas pelas questões que tratavam das técnicas adequadas de amamentação $(36,4 \%)$. Em um estudo realizado em Fortaleza, que também abordou esses quatro domínios temáticos, verificou-se que a pergunta de número um, acerca da prática de exercícios mamilares durante a gestação, inserida na primeira abordagem, foi a que apresentou maior prevalência de respostas erradas $(27,0 \%)^{(11)}$. Essa conduta de estímulo dos mamilos por meio dos exercícios é contraindicada durante a gestação, uma vez que as excitações da aréola e do mamilo induzem à produção de ocitocina, possível de acarretar contrações uterinas, que aumentam o risco de parto prematuro ${ }^{(12)}$.

Percebeu-se, com a intervenção educativa, aumento mais representativo no número de acertos das questões da abordagem quatro, acerca das técnicas adequadas de amamentação, o que também foi observado no estudo de Fortaleza ${ }^{(11)}$ previamente referido, cujas médias de acertos nas respostas, após a realização de palestras, atingiu 90,3\%. Esse achado é satisfatório, uma vez que a execução de uma pega correta da mama pelo lactente mantém adequada a produção de leite e protege o mamilo de fissuras ${ }^{(14)}$.

Outro estudo, realizado no distrito de Karnataka, na Índia, investigou conhecimentos, atitudes e práticas sobre as técnicas de amamentação de 118 mulheres na primeira semana pós-parto, antes e após treinamento baseado em imagens que mostravam erros e acertos no posicionamento de mãe e bebê durante $o$ ato de amamentar. Os autores identificaram melhora significativa nos conhecimentos e atitudes dessas mulheres após o programa de treinamento ${ }^{(23)}$. É importante ressaltar que a vivência do ato de amamentar pelas mulheres indianas, embora de uma semana ou menos, pode ter contribuído com a apreensão do conhecimento. Mas esses autores também concluíram que mulheres que foram educadas sobre a técnica de amamentar durante o pré-natal evoluíram de forma mais significativa, o que reforça a importância da abordagem multiprofissional permanente sobre aleitamento materno desde o início da gestação.

A segunda abordagem que mostrou maior impacto após a intervenção abordou os cuidados com as mamas antes do parto. Já no estudo de Fortaleza ${ }^{(11)}$ esse lugar foi ocupado pelo bloco temático que interrogou sobre as vantagens da amamentação para o bebê e para a mãe.

Um estudo de intervenção desenvolvido no Mississipi, com 29 mulheres que planejavam engravidar, que estavam grávidas ou que tinham filhos com idade inferior a um ano de idade, constituiu-se de sessões educativas que se concentraram no aumento do conhecimento e conscientização das mulheres sobre nutrição e atividade física durante o período pré-natal e pós-natal, prática de tabagismo, aleitamento materno, sono infantil seguro, saúde mental materna e redução de estresse. Seu objetivo, em longo prazo, era, no que tange à amamentação, a partir da melhoria do nível de conhecimento das mães, acentuar a prática e reduzir a mortalidade infantil. Foi detectado que 90,5\% das participantes identificaram com sucesso pelo menos quatro benefícios da prática de amamentação(24).

Em concordância, em um estudo realizado na Itália, com 503 mães de crianças de idade inferior a dois anos, identificou-se que a maioria das mulheres apresentavam conhecimentos adequados acerca dos benefícios do aleitamento materno exclusivo para a mãe e para a criança ${ }^{(25)}$. Na presente pesquisa, antes mesmo das ações educativas, percebeu-se maior conhecimento das mulheres nesse bloco temático em relação a todos os outros, o que sugere que as gestantes são bem informadas acerca dos benefícios do leite materno para o binômio mãe-filho, provavelmente não sendo a falta de conhecimento sobre esse aspecto um fator determinante da baixa prevalência da prática de aleitamento materno.

Um estudo transversal realizado em Recife, que avaliou o trabalho de 84 equipes de Saúde da Família (ESF) de 42 Centros de Saúde (CS), mostrou que, das 84 ESF, apenas seis $(7,1 \%)$ apresentavam ações de incentivo ao aleitamento materno efetivamente implantadas em suas rotinas. Esse achado grave evidencia o quanto o sistema 
público de saúde necessita evoluir para mudar o quadro brasileiro de baixa prática de aleitamento materno exclusivo ou até mesmo de aleitamento predominante ${ }^{(26)}$, pois as mulheres têm maior probabilidade de amamentar quando são incentivadas pelos profissionais de saúde. A postura profissional durante o pré-natal acerca da amamentação ainda é inadequada ${ }^{(3)}$, o que não ocorre apenas no Brasil.

O incentivo à prática do aleitamento e alimentação saudável para crianças menores de dois anos é estratégia fundamental para promoção da saúde de uma população, pois se reflete de forma eficaz no aumento da prevalência da prática e na redução da morbimortalidade infantil(13). Além disso, os impactos ultrapassam a fase da infância, já que uma alimentação saudável nesse período, pela teoria do imprinting metabólico, determinará a sua condição de saúde futura e, até mesmo, de seus descendentes ${ }^{(27)}$. Para as mães que amamentam, contribui com a prevenção de cânceres de mama e de ovário, obesidade, diabetes mellitus e hipertensão arterial(3).

Portanto, o exposto até aqui mostra que intervir sobre o aleitamento materno melhora conhecimentos e atitudes ${ }^{(19)}$, motiva as mulheres para o seu exercício ${ }^{(3)}$ e contribui com a promoção da saúde da saúde da população em longo prazo $^{(13)}$.

Essas ações devem, inicialmente, priorizar as comunidades de baixa renda, pois é mais elevada a prevalência de desmame precoce, com enfoque abrangente, que inclua os prejuízos decorrentes da introdução de alimentos não lácteos e do uso de mamadeiras ${ }^{(28)}$, e que também envolvam os pais no processo, para sucesso da prática ${ }^{(29)}$.

Contudo, é difícil ser um profissional de saúde que incentiva as mulheres para o aleitamento materno quando não se acredita nas vantagens dessa conduta. Tem-se evidência de que é comum que os médicos pediatras desacreditem que os benefícios da amamentação superam as dificuldades ou os inconvenientes que envolvem o ato ${ }^{(18)}$. Assim, aprimorar permanentemente as competências e habilidades dos profissionais de saúde para a promoção do aleitamento materno como atividade de rotina nas UAPS pode contribuir para a redução das posturas desestimuladoras da amamentação.

Por reconhecer isto, o Ministério da Saúde, através da Estratégia Amamenta e Alimenta Brasil, incentiva as secretarias de saúde dos municípios e do Distrito Federal, com suporte das secretarias estaduais de saúde, a criarem espaços coletivos para o desenvolvimento permanente de educação, formação e práticas crítico-reflexivas em saúde, que potencializem a qualidade do cuidado. As ações devem ser fundamentadas nas diretrizes das políticas nacionais de Atenção Básica, de Promoção da Saúde, de Alimentação e Nutrição, e de Promoção, Proteção e Apoio ao Aleitamento Materno ${ }^{(13)}$.

Em virtude da educação em saúde integrar o objeto de estudo deste trabalho, deve-se destacar que seus benefícios vão além dos apresentados para o público materno-infantil. A educação permanente de trabalhadores da saúde ${ }^{(7,13)}$ e a educação em saúde centrada na participação ativa de todos os sujeitos e no estabelecimento de vínculo entre o público assistido e os profissionais representam instrumentos de empoderamento e estímulo dos usuários de serviço de saúde para o autocuidado e, portanto, para a adoção de práticas que beneficiam a sua saúde e qualidade de vida. Desse modo, entende-se que educação e promoção da saúde caminham juntas ${ }^{(30)}$.

É relevante referir algumas limitações que podem ter comprometido a qualidade deste estudo: foi comum entre as gestantes o relato sobre a dificuldade em entender algumas perguntas, aparentemente elaboradas de forma confusa, apesar das explicações prestadas pelo pesquisador. As diferenças metodológicas entre os estudos de investigação de conhecimento de mulheres sobre aleitamento materno, desde o tamanho amostral, passando pelo ciclo de vida no qual as mulheres se encontravam (gestação, puerpério, lactação), até os domínios do conhecimento abordados nos questionários, dificultaram o diálogo desta pesquisa com as dos demais autores.

Por fim, é importante ressaltar a importância dos gestores de saúde, profissionais de saúde e, até mesmo, da academia ao exercerem sua função social, priorizarem as ações de incentivo ao aleitamento materno em suas rotinas nos serviços de saúde. Sugere-se a realização de mais estudos de intervenção no âmbito do aleitamento materno com as mulheres do município estudado, através dos quais elas sejam acompanhadas em longo prazo, com o intuito de se obter achados concretos acerca do impacto das estratégias educativas no prolongamento do tempo de aleitamento materno.

\section{CONCLUSÃO}

As gestantes investigadas apresentaram conhecimento adequado sobre a maioria dos questionamentos acerca do aleitamento materno desde o período préintervenção, principalmente quanto aos benefícios dessa prática para a mãe e para o bebê. Mas a educação em saúde mostrou-se eficaz em melhorar esses conhecimentos, o que sugere que se adequada e permanentemente realizada durante todo o pré-natal, poderá refletir em melhoria dos números associados à prática do aleitamento materno. 


\section{AGRADECIMENTOS E CONFLITOS DE INTERESSE}

Autores agradecem às gestantes participantes da pesquisa e aos profissionais da UAPS.

Os pesquisadores declaram não haver conflitos de interesses.

\section{CONTRIBUIÇÕES}

Andreza Kathiuze Maia contribuiu com a elaboração do projeto da pesquisa, coleta de dados, tabulação e análise de dados e com a redação do manuscrito. Luis Clenio Jário Moreira contribuiu com a análise de dados e a redação do manuscrito. Bruna Yhang da Costa Silva contribuiu com a elaboração do projeto, tabulação e análise de dados e a redação do manuscrito.

\section{REFERÊNCIAS}

1. Takushi SAM, Tanaka ACA, Gallo PR, Machado MAMP. Motivação de gestante para o aleitamento materno. Rev Nutr. 2008;21(5):491-502.

2. Zeleke LB, Gebremichael MW, Adinew YM, Gelaw KA. Appropriate weaning practice and associated factors among infants and young children in northwest Ethiopia. Int J Sport Nutr Exerc Metab. 2017;2017(8):1-7.

3. Schwarz EB, Nothnagle M. The maternal health benefits of breastfeeding. Am Fam Physician. 2015;91(9):603-4.

4. Motee A, Jeewon R. Importance of exclusive breast feeding and complementary feeding among infants. Curr Res Nutr Food Sci. 2014;2(2):56-72.

5. Ministério da Saúde (BR). II Pesquisa prevalência de aleitamento materno nas capitais brasileiras e Distrito Federal. Brasília (DF): Ministério da Saúde; 2009.

6. Amorim MM, Andrade ER. Atuação do enfermeiro no PSF sobre aleitamento materno. Perspect Online. 2009;3(9):93-110.

7. Ministério da Saúde (BR). Política nacional de promoção da saúde: anexo I da portaria de consolidação $n^{\circ} 2$, de 28 de setembro de 2017, que consolida as normas sobre as políticas nacionais de saúde do SUS. Brasília (DF): Ministério da Saúde; 2018.

8. Silva JAR, Lemos EC, Hardman CM, Santos SJ, Antunes MBC. Educação em saúde na estratégia de saúde da família: percepção dos profissionais. Rev Bras Promoç Saúde. 2015;28(1):75-81.

9. Triola MF. Introdução à estatística. $12^{\mathrm{a}}$ ed. Rio de Janeiro: LTC; 2017.

10. National Health Service England (UK). Physical activity guidelines for children and young people [Internet]. London: NHS; 2018 [acesso em 2019 Out 03]. Disponível em: https://www.nhs.uk/live-well/exercise/physicalactivity-guidelines-children-and-young-people/

11. Freitas GL, Joventino ES, Aquino PS, Pinheiro AKB, Ximenes LB. Evaluating the knowledge on breastfeeding among pregnant women. REME Rev Min Enferm. 2008;12(4):461-8.

12. Ministério da Saúde (BR). Saúde da criança: aleitamento materno e alimentação complementar. Brasília (DF): Ministério da Saúde; 2015.

13. Ministério da Saúde (BR). Estratégia nacional para promoção do aleitamento materno e alimentação complementar saudável no sistema único de saúde. Brasília (DF): Ministério da Saúde; 2015.

14. Vitolo MR. Nutrição: da gestação ao envelhecimento. $2^{\mathrm{a}}$ ed. Rio de Janeiro: Rubio; 2015.

15. Carvalho MR, Tavares LAM. Amamentação: bases científicas. $3^{a}$ ed. São Paulo: Guanabara Koogan; 2010.

16. Ministério da Saúde (BR), Sociedade Brasileira de Pediatria. Amamentação: muito mais que alimentar a criança [Internet]. Brasília (DF): Ministério da Saúde; c2009 [acesso em 2019 Out 17]. Disponível em: https:// www.sbp.com.br/departamentos-cientificos/aleitamento-materno/videos/

17. World Health Organization. Global strategy for infant and young child feeding. Geneva: WHO; 2002.

18. Meek JY, Hatcher AJ. The breastfeeding-friendly pediatric office practice. Pediatrics. 2017;139(5):e20170647. 
19. Lima TM, Osório MM. Perfil e fatores associados ao aleitamento materno em crianças menores de 25 meses da Região Nordeste do Brasil. Rev Bras Saúde Mater Infant. 2003;3(3):305-14.

20. Instituto Brasileiro de Geografia e Estatística. Pesquisa nacional por amostra de domicílios contínua: educação 2018. Rio de Janeiro: IBGE; 2019.

21. Cavalcante MCV, Lamy F Filho, França AKTC, Lamy ZC. Relação mãe-filho e fatores associados: análise hierarquizada de base populacional em uma capital do Brasil-estudo BRISA. Ciênc Saúde Colet. 2017;22(5)1683-93.

22. Brito RS, Oliveira EMF. Opinião do pai sobre o aleitamento materno. Rev RENE. 2006;7(1):9-16.

23. Tella K, Guruvare S, Hebbar S, Adiga P, Rai L. Knowledge, attitude, and practice of techniques of breastfeeding among of postnatal mothers in a coastal district of Karnataka. Int J Med Sci Public Health. 2016;5(1):28-34.

24. Fastring D, Mayfield-Johnson S, Madison J. Evaluation of a health education intervention to improve knowledge, skills, behavioral intentions and resources associated with preventable determinants of infant mortality. Divers Equal Health Care. 2017;14(6):290-5.

25. Cascone D, Tomassoni D, Napolitano F, Giuseppe G. Evaluation of knowledge, attitudes, and practices about exclusive breastfeeding among women in Italy. Int J Environ Res Public Health. 2019;16(1):2118-29.

26. Bezerra LCA, Fruas PG, Vidal SA, Macedo VC, Vanderlei LC. Breast-feeding: assessment of the implementation of a program at basic healthcare units in Recife, Pernambuco State (2002). Ciênc Saúde Colet. 2007;12(5):1309-17.

27. Mozetic RM, Silva SDC, Ganen AP. A importância da nutrição nos primeiros mil dias. Rev Eletrônica Acervo Saúde. 2016;8(2):876-84.

28. Akinyinka MR, Olatona FA, Oluwole EO. Breastfeeding knowledge and practices among mothers of children under 2 years of age living in a military barrack in Southwest Nigeria. Int J MCH AIDS. 2016;5(1):1-13.

29. Vijayalakshmi P, Susheela T, Mythili D. Knowledge, attitudes, and breast feeding practices of postnatal mothers: a cross sectional survey. Int J Health Sci (Qassim). 2015;9(4):364-74.

30. Janini JP, Bessler D, Vargas AB. Educação em saúde e promoção da saúde: impacto na qualidade de vida do idoso. Saúde Debate. 2015;39(105):480-90.

\section{Endereço do primeiro autor:}

Andreza Kathiuze Maia

Instituto Federal de Educação, Ciência e Tecnologia do Ceará - IFCE

Campus Limoeiro do Norte

Rua Estevam Remígio, 1145

Bairro: Monsenhor Otávio

CEP: 62930-000 - Limoeiro do Norte - CE - Brasil

E-mail: kathiuze_maia@hotmail.com

\section{Endereço para correspondência:}

Bruna Yhang da Costa Silva

Instituto Federal de Educação, Ciência e Tecnologia do Ceará - IFCE

Campus Limoeiro do Norte

Rua Estevam Remígio, 1145

Bairro: Monsenhor Otávio

CEP: 62930-000 - Limoeiro do Norte - CE - Brasil

E-mail: bruna.yhang@ifce.edu.br

Como citar: Maia AK, Silva BYC, Moreira LCJ. Eficácia de intervenções educativas com gestantes sobre o grau de conhecimento em aleitamento materno. Rev Bras Promoç Saúde. 2019;32:9001. 